\title{
Guidance and Control Approaches that Enable Titan Aerogravity Assist for an Enceladus Mission
}

Primary Author: Benjamin Tackett

Analytical Mechanics Associates

NASA Langley Research Center

Phone: 757-864-3894

E-Mail: benjamin.m.tackett@nasa.gov

Co-Authors: Richard W. Powell ${ }^{1}$, Soumyo Dutta ${ }^{2}$, Rafael A. Lugo ${ }^{2}$, Daniel Engel ${ }^{3}$

Endorsers:

James O. Arnold ${ }^{1}$

Aaron M. Brandis ${ }^{1}$

Hans Seywald ${ }^{1}$

Rohan G. Deshmukh ${ }^{1}$

Jeremy D. Shidner ${ }^{1}$

David M. Cornelius ${ }^{1}$
Ashley M. Korzun ${ }^{2}$

Alicia M. Dwyer Cianciolo ${ }^{2}$

Karl T. Edquist ${ }^{2}$

Paul V. Tartabini ${ }^{2}$

Brett R. Starr ${ }^{2}$

Min $\mathrm{Qu}^{2}$

${ }^{1}$ Analytical Mechanics Associates, Hampton VA

${ }^{2}$ NASA Langley Research Center, Hampton VA

${ }^{3}$ University of Illinois, Urbana-Champaign IL

${ }^{4}$ NASA Johnson Space Center, Houston TX

${ }^{5}$ Independent Consultant

${ }^{6}$ NASA Ames Research Center, Mountain View CA 


\section{Executive Summary}

A dedicated mission to Enceladus has been a major objective for past Planetary Decadal Surveys, but the mission requirements for Enceladus orbits or flybys due to $\Delta \mathrm{V}$ required for such scenarios is exorbitant. This white paper will describe how the use of advanced Guidance \& Control (G\&C) techniques could enable successful aerogravity assist maneuvers at Titan using existing low lift-to-drag ratio (L/D) vehicles, such as the Apollo capsule or Mars Science Laboratory shapes, and allow an orbiter to enter a Saturncentered orbit with flybys of Enceladus. Modern guidance algorithms and control methods have been shown to enable aerocapture maneuvers at Neptune and Uranus using low L/D vehicles, and these advancements can be leveraged to enable aerodynamic orbital transfers at the Gas Giants as well. The primary difference between aerogravity assist and aerocapture is that aerocapture leaves the vehicle in an elliptical orbit while an aero gravity assist would leave the vehicle in a hyperbolic orbit. This paper discusses the G\&C techniques, the current state of these methods, and how their use can enable a future mission to Enceladus.

\section{Introduction}

The 2009 paper by Spilker et al. [1] studied a variety of Enceladus mission architectures and determined that an aerogravity assist maneuver, where the atmospheric drag and gravity of a planet-sized body are used to provide the $\Delta \mathrm{V}$ and turn angle needed for an orbit insertion about another celestial object, would be best suited for an Enceladus mission. The proposed mission in that paper using aerogravity assist suggested Titan as the planetary body whose atmosphere and gravity could be used to decrease the velocity of the interplanetary trajectory to attain a Saturn capture orbit with flybys to study Enceladus. Titan is the lowest risk destination for an aero-assist maneuver due to low variability in high altitude atmosphere, as determined by the science provided by the Cassini Equinox Mission, and the large density scale height associated with Titan's atmosphere, which provides large altitude ranges where aero-assist maneuvers could be performed without interaction with terrain. The aerogravity assist maneuver being investigated would involve using the atmosphere of Titan to provide the required $\Delta \mathrm{V}$ to control the exit energy and turn angle for the Saturn capture orbit, while also providing $\Delta \mathrm{V}$ required to remove out-of-plane errors. This orbit can then be adjusted to intercept Enceladus and transfer to the desired science orbit.

Aerogravity assists have been studied for many years. However most, if not all, the studies only solved the in-plane dynamics. With the advent of new control strategies such as Direct Force Control (DFC) and new guidance algorithms, both the in-plane and out-of-plane errors can be controlled simultaneously, leading to a final target orbit. Additionally, these guidance and control techniques leverage development in this field over the last decade, which means that there is large continuity with the current state-of-theart.

\section{Concept of Operations}

The aerogravity assist maneuver consists of a combination of two more commonly known planetary maneuvers: gravity assist, where the gravity of a planetary body is used to turn the trajectory of a vehicle and change the relative speed with respect to another body; and aerocapture, where the aerodynamic forces within the atmosphere of a planetary body are used to reduce the incoming interplanetary trajectory velocity and attain capture orbit velocities. Figure 1 shows the concept of operations for the aerogravity assist maneuver to attain a Saturn orbit while utilizing the gravity and atmosphere of Titan to reduce the interplanetary velocity. The spacecraft approaches Titan in a hyperbolic orbit with respect to both Titan and Saturn. The vehicle has a periapsis that is inside Titan's atmosphere and while it is within Titan's atmosphere, aerodynamic drag produces $\Delta \mathrm{V}$ that reduces the speed of the vehicle. Unlike aerocapture, the 
spacecraft exits Titan's atmosphere still at a hyperbolic trajectory, but is at an energy state that ensures the desired Saturn orbit. Once the spacecraft reaches a desired phasing maneuver location, an impulsive
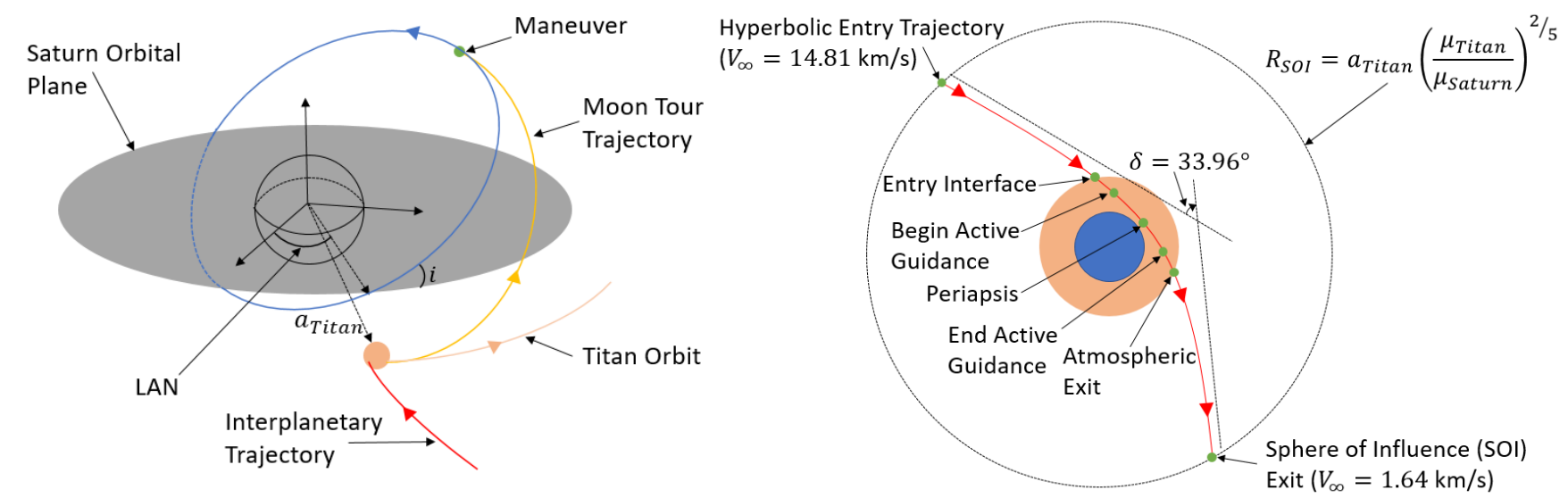

Figure 1. (left) Saturn-centered view of aerogravity assist maneuver, (right) Titan-centered view of aerogravity assist maneuver

burn is performed to reach the final target Saturn orbit, which in this case will conduct flybys of Enceladus.

The mission architecture described in this white paper assumes that the spaceflight trajectory to the Saturnian system has already been determined such that the vehicle intercepts Titan for the purpose of performing an aerogravity assist maneuver. The aerogravity assist will provide the majority of the $\Delta \mathrm{V}$ required to transfer the vehicle from a hyperbolic trajectory to a captured orbit about Saturn which is then followed by a series of small propulsive transfers to adjust the vehicle into the desired science orbit. Without aerogravity assist, the spacecraft would need more than $12 \mathrm{~km} / \mathrm{s}$ propulsive $\Delta \mathrm{V}$ to attain the desired capture orbit; with aerogravity assist, which requires a heatshield and atmospheric $\mathrm{G} \& \mathrm{C}$, the final propulsive correction $\Delta \mathrm{V}$ will be of the order $100 \mathrm{~m} / \mathrm{s}$.

To perform the aerogravity assist, a numerical predictor-corrector (NPC) algorithm will guide the vehicle based on an initial reference trajectory using repeated predictions of the remaining trajectory. These guidance techniques are similar to those being considered for human return from the Moon and Mars and has been demonstrated in Exploration Flight Test 1 (EFT1) mission in 2014. The control method investigated in this white paper will be DFC. For aerocapture three propulsive transfers (periapsis correction, apoapsis correction, and wedge angle correction) are used to adjust an aerocapture exit orbit to the desired science orbit; however, for aerogravity assist, the propulsive maneuvers will include a spindown from the aerogravity assist exit orbit to an orbit about Saturn with an intercept at Enceladus and a propulsive correction of the residual out of plane error.

A companion paper to this white paper discusses the vehicle design aspects of a Titan aerogravity assist mission in order to enable observation of Enceladus [2]. That paper focuses on the Thermal Protection System (TPS) options available for aerogravity assist and discusses some of the potential vehicle design characteristics for six design trajectories that attain a direct (D) Enceladus orbit or attain a Saturn-centered orbit for Moon tours (MT). Table 1 reproduces the summary of the trajectories from the companion paper. For this paper, the focus of the G\&C work was with the February 23, 2043 Moon Tour trajectory, which reaches Titan on that day and has the highest arrival hyperbolic excess velocity $(14.81 \mathrm{~km} / \mathrm{s})$ and a relatively low required outbound Titan-centric hyperbolic excess velocity $(1.64 \mathrm{~km} / \mathrm{s})$, all the while attaining a turn angle of $33.96 \mathrm{deg}$. 
Table 1. Summary of six potential Titan aerogravity assist trajectories for Enceladus observation [2]

\begin{tabular}{|c|c|c|c|c|c|c|c|c|c|c|c|c|}
\hline $\begin{array}{c}\text { Arrival } \\
\text { Date }\end{array}$ & $\begin{array}{l}\mathbf{V}_{\infty} \text { in } \\
\mathbf{k m} / \mathbf{s}\end{array}$ & $\begin{array}{c}\mathbf{V}_{\infty} \\
\text { out } \\
\mathbf{k m} / \mathbf{s}\end{array}$ & $\underset{\text { Deg. }}{\Delta_{\text {DGA }} \text { AGA }}$ & $\begin{array}{l}\text { DV, } \\
\text { km/s to } \\
\text { Encel- } \\
\text { Adus }\end{array}$ & $\begin{array}{c}\text { L/D } \\
\text { (Const- } \\
\text { ant) }\end{array}$ & $\begin{array}{c}\text { EFPA } \\
\text { Inertial } \\
\text { Deg. }\end{array}$ & $\begin{array}{c}\text { Peak } \\
\text { stag. } \\
\text { Press. } \\
\text { Pascals }\end{array}$ & $\begin{array}{c}\text { Peak } \\
\text { Conv. } \\
\text { heat } \\
\text { rate, } \\
\text { W/cm }{ }^{2}\end{array}$ & $\begin{array}{c}\text { Peak } \\
\text { Rad. } \\
\text { heat } \\
\text { rate, } \\
\text { W/cm }{ }^{2}\end{array}$ & $\begin{array}{c}\text { Peak } \\
\text { Tot. } \\
\text { heat } \\
\text { rate, } \\
\text { W/cm } \\
\end{array}$ & $\begin{array}{c}\text { Tot. } \\
\text { heat } \\
\text { Load, } \\
\text { J/cm² }\end{array}$ & $\begin{array}{c}\text { Atm. } \\
\text { Flight } \\
\text { Time, } \\
\text { Sec. }\end{array}$ \\
\hline $\begin{array}{c}2 / 11 / 43 \\
\text { (D) }\end{array}$ & 11.7 & 3.3 & 14.3 & 3.9 & -0.0168 & -36.708 & $1.78^{+4}$ & 200 & 327 & 523 & 35153 & 738 \\
\hline $\begin{array}{c}2 / 13 / 43 \\
\text { (D) }\end{array}$ & 7.3 & 1.197 & 47.16 & 5.47 & -0.0544 & -35.907 & $6.72^{+3}$ & 98 & 35 & 117 & 21784 & 1262 \\
\hline $\begin{array}{c}2 / 23 / 43 \\
\text { (D) }\end{array}$ & 14.80 & 2.6 & 18.5 & 3.8 & -0.0114 & -37.223 & $3.22^{+4}$ & 411 & 2286 & 2697 & 119868 & 792 \\
\hline $\begin{array}{c}2 / 13 / 43 \\
\text { (MT) }\end{array}$ & 7.3 & 1.252 & 45.633 & 0.618 & -0.0534 & -35.904 & $6.71^{+3}$ & 98 & 34 & 117 & 21787 & 1249 \\
\hline $\begin{array}{c}2 / 11 / 43 \\
\text { (MT) }\end{array}$ & 11.3 & 1.64 & 23.0 & 0.18 & 0.0856 & -37.786 & $2.19^{+4}$ & 199 & 270 & 461 & 27990 & 947 \\
\hline $\begin{array}{c}2 / 23 / 43 \\
\text { (MT) }\end{array}$ & 14.81 & 1.64 & 33.96 & 0.17 & -0.0387 & -37.134 & $3.22^{+4}$ & 410 & 2276 & 2686 & 120538 & 988 \\
\hline
\end{tabular}

Due to the complexities of this trajectory for G\&C aspects of flight, this trajectory was chosen as the example for this paper. With a focus on G\&C aspects of Titan aerogravity assist, this paper does not consider structural sizing, TPS design, and packaging constraints. These topics are covered in greater detail in the companion paper [2]; however, the specific development of the G\&C methods that enable the aerogravity assist maneuver and in turn enable missions that conduct Enceladus observations is the crux of this paper. For the analysis of this paper, the focus is on the usage of heritage entry system configurations, and the example used here is the $70 \mathrm{deg}$. sphere-cone Mars Science Laboratory shape. The G\&C methods would work with other existing shapes, such as the Apollo capsule or other sphere-cones used for planetary entry, such as the Genesis sample return probe (60 deg. sphere-cone) or Pioneer Venus probes (45 deg. sphere-cone), with some tuning of the G\&C parameters.

\section{Advancements in Guidance \& Control}

Guidance Schemes

In the past two decades, several advancements in the guidance and controls fields for planetary entry missions make maneuvers such as aerogravity assists attainable. One such development is the continued development of numerical predictor-corrector (NPC) guidance schemes. A NPC is defined as a class of algorithms created to integrate ordinary differential equations in two steps (prediction and correction) to determine an unknown function that satisfies a given differential equation. When using NPCs for guidance algorithms, a trajectory is generated and split into segments where the predictor corrector will create a set of controls such that the constraints for each segment are satisfied. Predictor corrector algorithms in general achieve this by generating a predicted trajectory given the current vehicle state and control vector for a given segment. The trajectory is compared against the constraints and the algorithm calculates the required changes in the control vector to minimize the errors for each segment before calling the algorithm again. Guidance schemes using NPCs have been recently utilized for studying humans to Mars trajectories [3], Ice Giants aerocapture [4], and are now being used to study aerogravity assist maneuvers at Titan.

The general characteristics of NPC schemes and the difference between numerical and analytical methods is the numerical propagation of the trajectory to create a prediction of the target condition, and guided steps to amend the parameters of the design, reducing errors in the target conditions. The predictorcorrector method creates a guidance system which can design a trajectory and provide adaptive guidance 
commands to the vehicle without using a pre-stored trajectory. The ability to adapt the guidance without relying on a pre-stored trajectory allows the vehicle to handle off-nominal conditions and larger dispersions which would be more severe than those a reference or terminal point following scheme could handle.

\section{Control Methods}

Traditionally, control mechanisms used during hypersonic entry missions have consisted of bank angle modulation and reaction control system thrusters. These mechanisms have been successfully used by the Space Shuttle and the Mars Science Laboratory; however, new

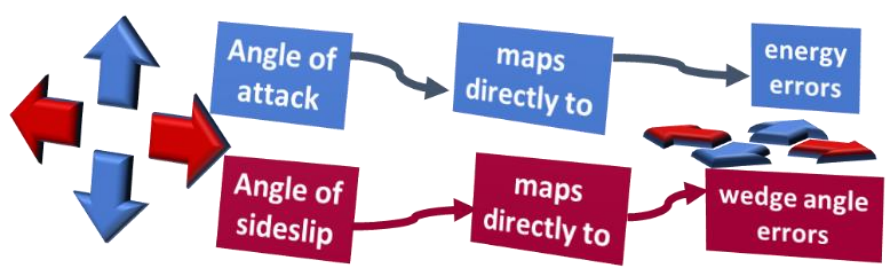

Figure 2. Application of Direct Force Control [4] methods, like DFC, when paired with the previously mentioned guidance schemes can allow independent control of longitudinal and lateral motion of the spacecraft, as shown in Figure 2, and provide greater precision and accuracy. This method of control involves modulating the aerodynamics of the vehicle inflight to control the angle of attack and sideslip angle of the vehicle. These two flight parameters alter the aerodynamic forces on the vehicle to control the in-plane trajectory and the out-of-plane errors during an aero-assist maneuver. This type of control is very useful for decoupling the control of final orbital energy and wedge angle.

In the past, flaps have been used to create this type of control authority during flight. This can be seen in practice by the NASA Space Shuttle during re-entry allowing for much more precise landings than those achieved by uncontrolled capsules. Flaps are also used on both commercial and private flight vehicles, proving to be a reliable form of control for many years.

Many previous NASA missions have also shifted the center-of-gravity (CG) to modify the aerodynamics of a vehicle during flight. The $\mathrm{CG}$ shift has been used to alter the trim angle of attack of the vehicle by jettisoning mass from a point near the edge of the vehicle thereby inducing a trim angle of attack and altering the aerodynamics of the vehicle. Following the induced trim, the vehicle follows a bank angle control scheme, which couples the in-plane and outof-plane errors of the trajectory creating a more complex guidance requirement. Next, additional mass is ejected, which shifts the $\mathrm{CG}$ and returns the trim angle of attack to zero for the final phase of flight. While this method is used primarily for the minimal complexity associated with the jettison, it has inefficiencies in reducing available mass for the

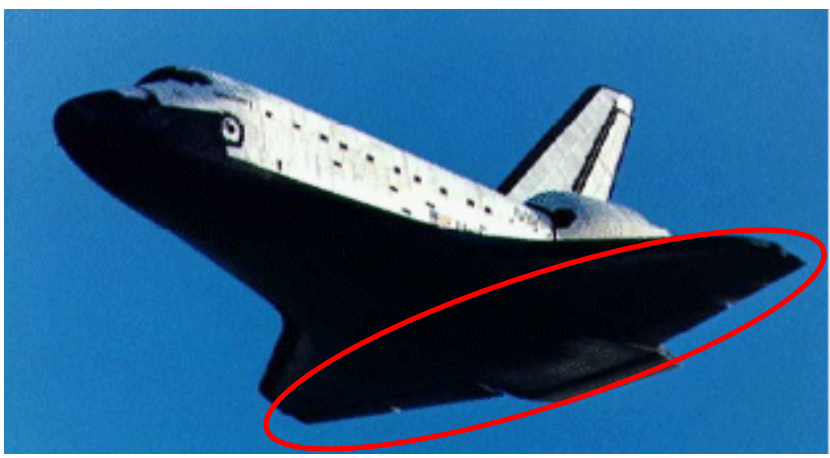

Figure 3. NASA Space Shuttle, Public-use Image

\section{Jettison two $75 \mathrm{~kg}$ Tungsten Weights}

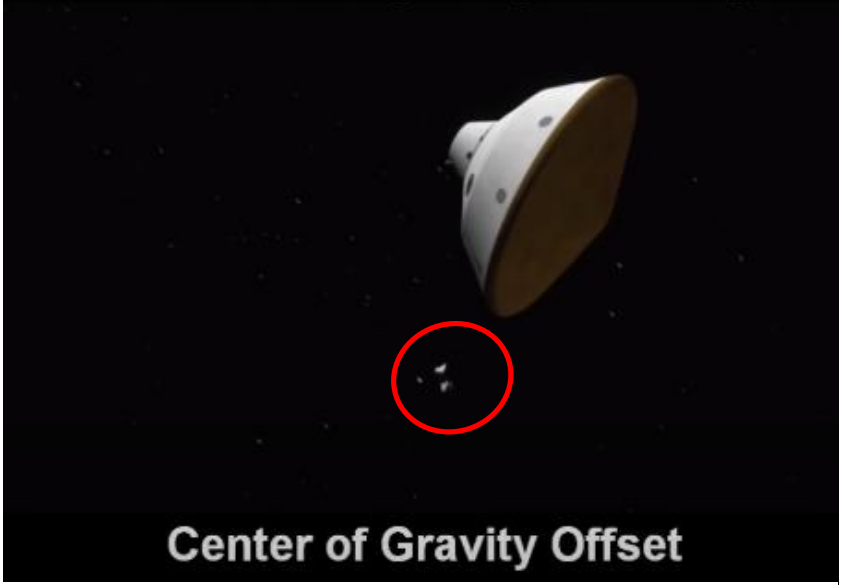

Figure 4. Mars Science Laboratory CG shift [10] science payload and coupling the in-plane and out-of-plane errors. 


\section{Results of Aerogravity Assist Maneuvers}

Figure 5a shows the Titan-centered, aerogravity assist trajectory for the aforementioned Moon tour scenario using an NPC based guidance scheme and a DFC controller. For the aeroassist trajectory, the spacecraft dips into the Titanian atmosphere but stays well above the terrain at a minimum altitude of around $200 \mathrm{~km}$; it then decelerates by an equivalent $\Delta \mathrm{V}$ of $1.25 \mathrm{~km} / \mathrm{s}$ before exiting the atmosphere. Figure 5b shows the maximum deceleration is in the range of 15 Earth g's, making the trajectory amenable to a large variety of scientific instrumentations, while Figure 5c shows the specific energy change during the aerogravity assist maneuver for Saturn orbit is achieved in just $\sim 5$ minutes versus traditional all-propulsive orbit insertion techniques which would take tens-of-minutes and a very large amount of propellant. Finally, Figure 5d shows the lift-to-drag ratios required during the aeroassist gravity maneuver to attain the target states. These L/D ratios are attainable by existing, heritage entry configurations like spherecones and capsules, and hence precludes the need for development of a new class of vehicles for Titan aerogravity assist.

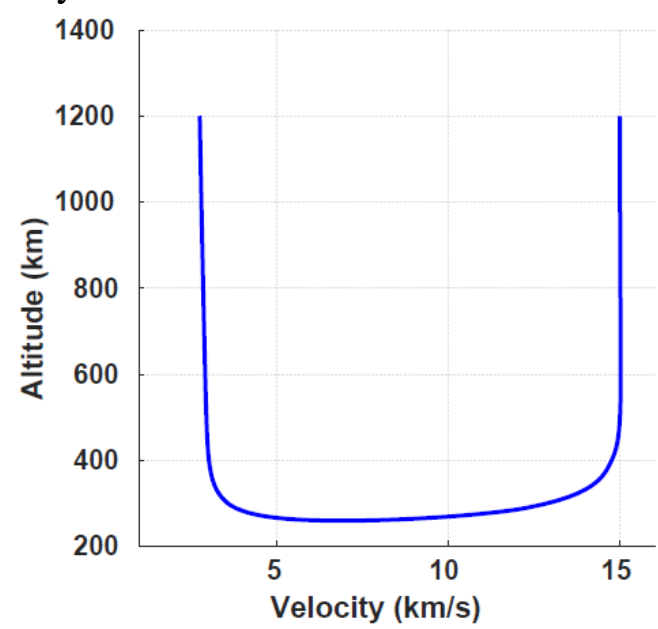

(5a) Altitude vs. Velocity

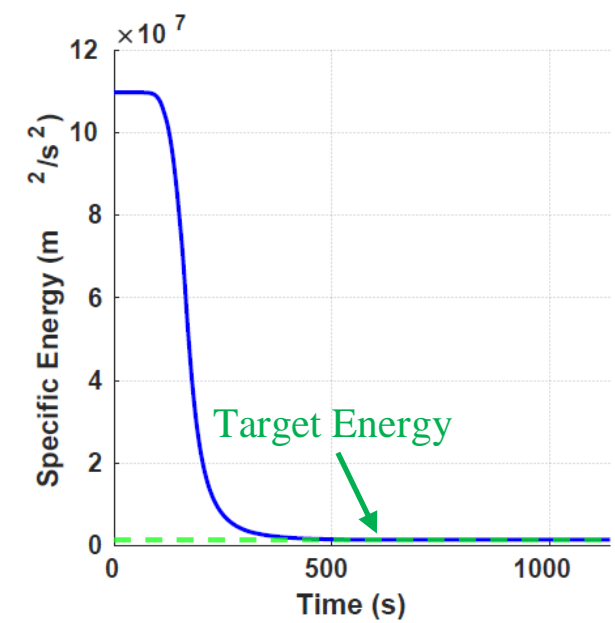

(5c) Specific energy

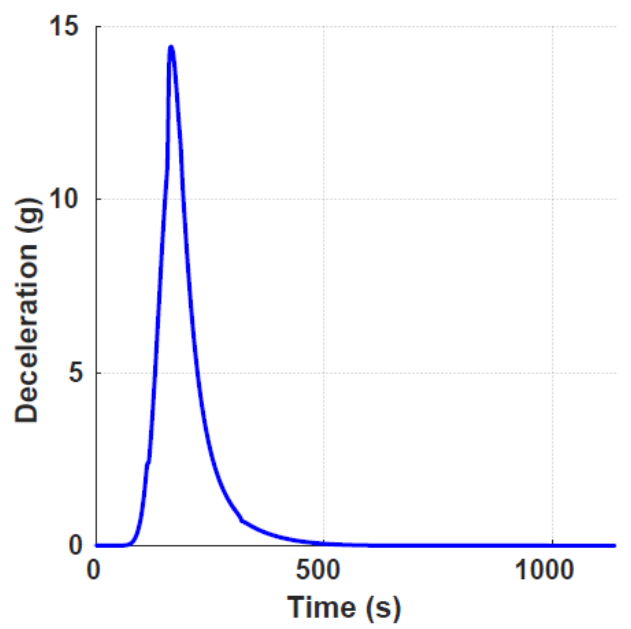

(5b) Time History of Deceleration

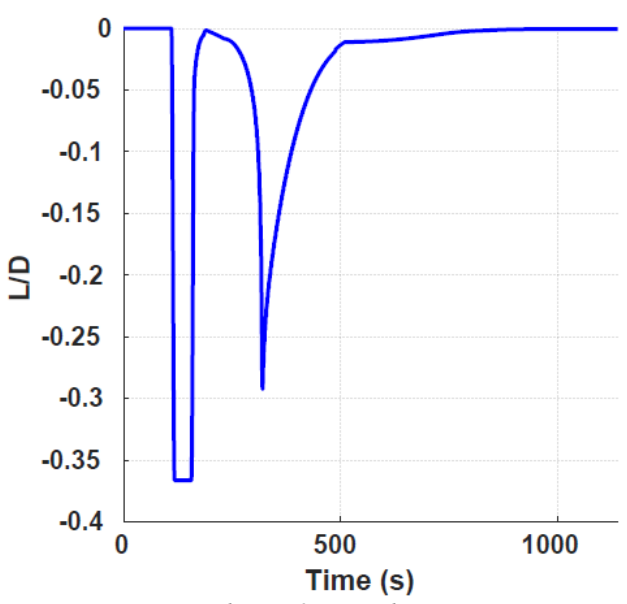

(5d) Lift-to-drag ratio

Figure 5. Titan Aerogravity assist trajectory for the 2/23/43 Moon Tour scenario 
The aerogravity assist maneuver reduces the necessary propellant load needed for a Saturn capture orbit to observe Enceladus by $99.5 \%$ as shown in Table 2. The mass savings from the $\Delta \mathrm{V}$ difference is not completely similar, as the aerogravity assist maneuver does require TPS material and heatshield structure, but past studies by Spilker et. al [1] and the companion paper to this white paper shows that the TPS mass fraction is a substantially smaller fraction than the propellant needed for a traditional, all-propulsive case.

Table 2. $\Delta \mathrm{V}$ differences between all-propulsive and aerogravity assist case

\begin{tabular}{|l|l|}
\hline Maneuver & Value \\
\hline All-Propulsive Maneuver $\Delta \mathrm{V}$ & $12200 \mathrm{~m} / \mathrm{s}$ \\
\hline Total Correction $\Delta \mathrm{V}$ for Aerogravity & $53.54 \mathrm{~m} / \mathrm{s}$ (orbital energy correction) \\
Assist (NPC \& DFC) & $5.34 \mathrm{~m} / \mathrm{s}$ (wedge angle correction) \\
\hline
\end{tabular}

\section{Conclusions}

Enceladus has been of keen interest to the scientific community, but a dedicated mission to the moon requires substantial spacecraft capability to achieve specific science orbits. Spilker, et al. [1] have shown that an aerogravity assist maneuver at Titan using heritage entry shapes is an enabling technology for Enceladus exploration. This white paper provides an assessment of the aerogravity assist scenario presented in [1] and discusses development of modern guidance and control schemes that enable a feasible aerogravity assist maneuver. This white paper investigates an example of a potentially stressing moon tour trajectory where aerogravity assist can reduce the propellant requirements for orbital insertion by $99.5 \%$ compared to traditional, all-propulsive maneuvers. Together with the companion paper [2], this white paper shows that there is a pathway to conducting aerogravity assist maneuvers at Titan using modern and heritage technologies, opening the doors to exploring scientifically compelling destinations like Enceladus.

\section{Recommendations}

This white paper has presented a preliminary analysis of G\&C methods on the performance of a conceptual Enceladus science mission with a Titan aerogravity assist maneuver and has shown the potential propellant savings achievable through such methods. The companion white paper [2] provides a preliminary TPS analysis for a Titan aerogravity assist vehicle, rounding out another concern with such maneuvers. Given the significant potential of a Titan aerogravity assist maneuver in exploring the moons of Saturn, it is recommended that a detailed systems study be established as a method of maturing this concept and to further explore the aspects of this maneuver. The goals of this extended analysis are as follows: to thoroughly evaluate the impact of recent $\mathrm{G} \& \mathrm{C}$ advancements on mission performance for a science mission using an aerogravity assist maneuver, determine the complete thermal protection system requirements and available options for such a mission, perform a systems level design of a vehicle suited for this mission, and verify the hypotheses put forth in these partner white papers. Additionally, this extended study would provide an outline for steps with which aerogravity assist technology should be matured for use in a future Enceladus science mission. 


\section{References}

[1] T. Spilker and et al., "Analysis of Architectures for the Scientific Exploration of Enceladus," IEEEAC paper \#1644 2009 IEEE Aerospace Conference, March 2009.

[2] J. Arnold and et al., "Heatshields for Aerogravity Assist Vehicles Whose Deceleration at Titan," White Paper Submission for Planetary Science Decadal Survey, 2023 - 2032, 2020.

[3] R. Lugo and et al., "Overview of a Generalized Numerical Predictor-Corrector Targeting Guidance with Application to Human-Scale Mars Entry, Descent, and Landing," in Scitech 2020 Forum, 2020.

[4] B. Tackett and et al., "Investigation of Aerocapture Guidance \& Control for Ice Giants Missions," in International Plantary Probe Workshop (IPPW), Oxford, UK, 2019.

[5] P. Lu and et al., "Verification of a Fully Numerical Entry Guidance Algorithm," AIAA 2016-0377, 2016.

[6] D. Matz and et al., "Application of a Fully Numerical Guidance to Mars Aerocapture," 10.2514/6.2017-1901, 2017.

[7] S. Striepe and et al., "Program to optimize simulated trajectories (POST II)," (fourth ed.), Utilization Manual, vol. II, November 2017.

[8] R. Deshmukh and et al., "Investigation of Direct Force Control for Planetary Aerocapture at Neptune," Acto Astronautica, vol. 175, pp. 375-386, 2019.

[9] S. Dutta and et al., "Aerocapture as a Mission Option for Ice Giants Missions," in Workshop on In Situ Exploration of the Ice Giants, Marseille, France, 2019.

[10] Mars Science Laboratory - Entry, Descent \& Landing - Full Animation. [Film]. United States of America: NASA / JPL, 2012.

[11] M. Lockwood and et al., "Aerocapture Systems Analysis for a Titan Mission," NASA TM-2006214273.

[12] C. Heidrich and et al., "Modern Aerocapture Guidance to Enable Reduced-Lift Vehicles at Neptune," AAS 19-221, 2019.

[13] G. Walberg, "A Survey of Aeroassisted Orbit Transfer," J. Spacecr Rockets, 1985. 